\title{
PSLQ: An Algorithm to Discover Integer Relations
}

\author{
David H. Bailey* and J.M. Borwein ${ }^{\dagger}$
}

April 3, 2009

1. Introduction. Let $x=\left(x_{1}, x_{2}, \cdots, x_{n}\right)$ be a vector of real or complex numbers. $x$ is said to possess an integer relation if there exist integers $a_{i}$, not all zero, such that

$$
a_{1} x_{1}+a_{2} x_{2}+\cdots+a_{n} x_{n}=0
$$

By an integer relation algorithm, we mean a practical computational scheme that can recover the vector of integers $a_{i}$, if it exists, or can produce bounds within which no integer relation exists. As we will see in the examples below, an integer relation algorithm can be used to recognize a computed constant in terms of a formula involving known constants, or to discover an underlying relation between quantities that can be computed to high precision. At the present time, the most effective algorithm for integer relation detection is the "PSLQ" algorithm of mathematician-sculptor Helaman Ferguson [10, 4]. Some efficient "multi-level" implementations of PSLQ, as well as a variant of PSLQ that is well-suited for highly parallel computer systems, are given in [4].

PSLQ constructs a sequence of integer-valued matrices $B_{n}$ that reduces the vector $y=x B_{n}$, until either the relation is found (as one of the columns of $B_{n}$ ), or else precision is exhausted. At the same time, PSLQ generates a steadily growing bound on the size of any possible relation. When a relation is found, the size of smallest entry of the vector $y$ abruptly drops to roughly "epsilon" (i.e. $10^{-p}$, where $p$ is the number of digits of precision). The size of this drop can be viewed as a "confidence level" that the relation is real and not merely a numerical artifact - a drop of 20 or more orders of magnitude almost always indicates a real relation (see Figure 1).

Very high precision arithmetic must be used in PSLQ. If one wishes to recover a relation of length $n$, with coefficients of maximum size $d$ digits, then the input vector $x$ must be specified to at least $n d$ digits, and one must employ $n d$-digit floating-point arithmetic. Maple and Mathematica include multiple precision arithmetic facilities and Maple ships with a full implementation of PSLQ. One may also use any of several freeware multiprecision software packages, for example the ARPREC package by the first author

*Lawrence Berkeley National Laboratory, Berkeley, CA 94720, dhbailey@1bl.gov. Supported in part by the Director, Office of Computational and Technology Research, Division of Mathematical, Information, and Computational Sciences of the U.S. Department of Energy, under contract number DE-AC02$05 \mathrm{CH} 11231$.

†School of Mathematical and Physical Sciences, University of Newcastle, Callaghan, NSW 2308, Australia jonathan. borwein@newcastle.edu. au 


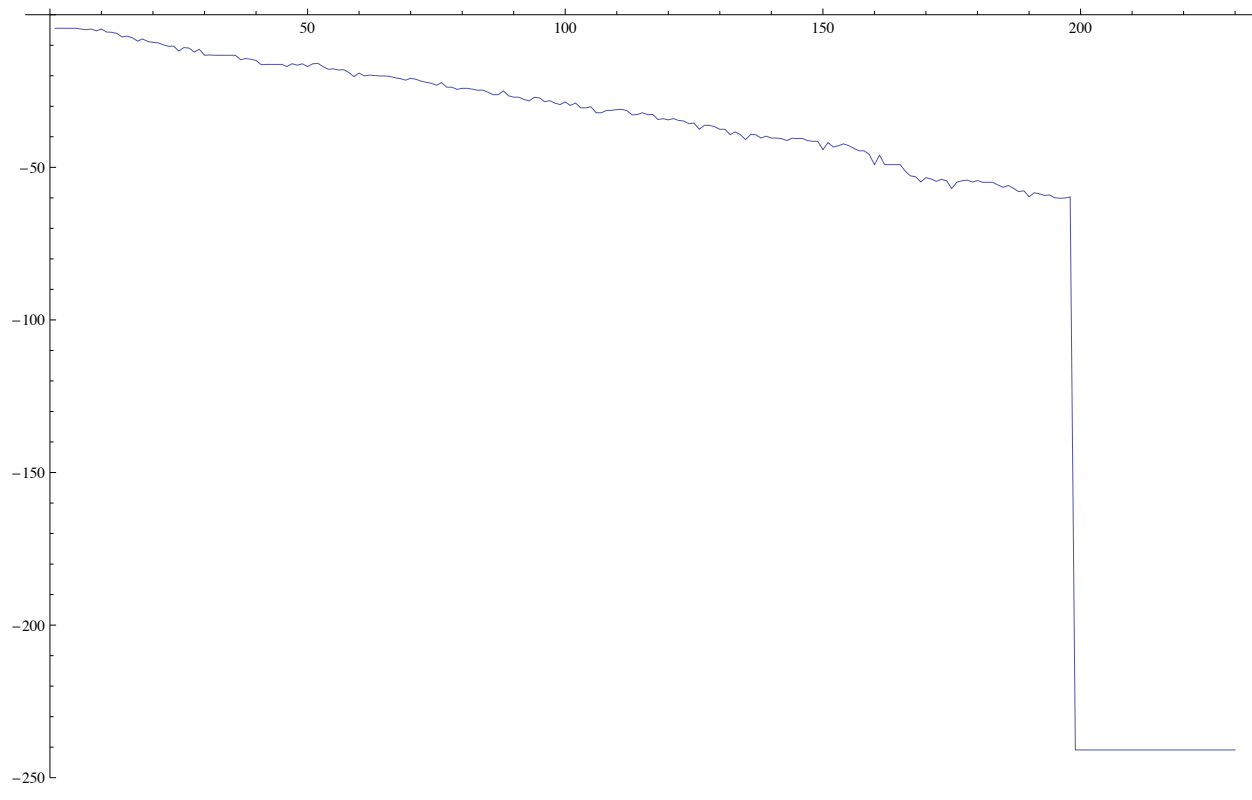

Figure 1: $\log _{10} \max _{k}\left|y_{k}\right|$ versus iteration number in a typical PSLQ run

and colleagues at LBNL [7]. In the remaining sections we describe various representative applications of PSLQ. More detail about these examples is given in [8] and the references therein.

2. Finding Algebraic Relations Using PSLQ. One immediate and impressive application of PSLQ in the field of mathematical number theory is to determine whether or not a given constant $\alpha$, whose value can be computed to high precision, is algebraic of some degree $n$ or less. This can be done by first computing the vector $x=\left(1, \alpha, \alpha^{2}, \cdots, \alpha^{n}\right)$ to high precision and then applying an integer relation algorithm to the resulting $(n+1)$-long vector. If a relation is found for $x$, then this relation vector is precisely the set of integer coefficients of a polynomial satisfied by $\alpha$ (to the precision specified).

One of the first results of this sort was the identification of the constant $B_{3}=$ $3.54409035955 \ldots B_{3}$ is the third bifurcation point of the logistic map $x_{k+1}=r x_{k}\left(1-x_{k}\right)$, which exhibits period doubling shortly before the onset of chaos. To be precise, $B_{3}$ is the smallest value of the parameter $r$ such that successive iterates $x_{k}$ exhibit eight-way periodicity instead of four-way periodicity. $B_{3}$ can be computed to arbitrarily high precision by means of an iterative algorithm [6]. When PSLQ is applied to the 13-long vector $\left(1, B_{3}, B_{3}^{2}, B_{3}^{3}, \cdots, B_{3}^{12}\right)$, one obtains the result that $B_{3}$ is a root of the polynomial

$$
\begin{aligned}
0= & 4913+2108 t^{2}-604 t^{3}-977 t^{4}+8 t^{5}+44 t^{6}+392 t^{7}-193 t^{8}-40 t^{9} \\
& +48 t^{10}-12 t^{11}+t^{12}
\end{aligned}
$$

Recently, $B_{4}=3.564407268705 \cdots$, the fourth bifurcation point of the logistic map, was identified using PSLQ by British physicist David Broadhurst [4]. Some conjectural reasoning had suggested that $B_{4}$ might satisfy a 240-degree polynomial, and some further analysis had suggested that the constant $\alpha=-B_{4}\left(B_{4}-2\right)$ might satisfy a 120 -degree 
polynomial. In order to test this hypothesis, Broadhurst applied a PSLQ program to the 121-long vector $\left(1, \alpha, \alpha^{2}, \cdots, \alpha^{120}\right)$. Indeed, a relation was found, though 10,000-digit arithmetic was required. The recovered integer coefficients descend monotonically from $257^{30} \approx 1.986 \times 10^{72}$ to 1 . This was subsequently proven using Groebner bases [6].

3. A New Formula for Pi. Through the centuries mathematicians have assumed that there is no shortcut to computing just the $n$-th digit of $\pi$. Thus, it came as no small surprise when such an algorithm was discovered in 1996 [3]. In particular, this simple scheme allows one to compute binary or hexadecimal (base-16) digits of $\pi$ starting at an arbitrary position, without computing any of the preceding digits. For instance, the one millionth hex digit of $\pi$ can be computed in this manner on a current-generation personal computer in only about 10 seconds run time. This scheme is based on the following new formula, which was discovered in 1996 using PSLQ:

$$
\pi=\sum_{k=0}^{\infty} \frac{1}{16^{k}}\left(\frac{4}{8 k+1}-\frac{2}{8 k+4}-\frac{1}{8 k+5}-\frac{1}{8 k+6}\right) .
$$

Since 1996, numerous formulas of this same type have been found for various constants $[1,8]$. Here, for instance, is a formula for $\pi^{2}$ that permits base- 3 digits to be computed beginning at an arbitrary starting position:

$$
\begin{aligned}
\pi^{2}= & \frac{2}{27} \sum_{k=0}^{\infty} \frac{1}{729^{k}}\left(\frac{243}{(12 k+1)^{2}}-\frac{405}{(12 k+2)^{2}}-\frac{81}{(12 k+4)^{2}}-\frac{27}{(12 k+5)^{2}}\right. \\
& \left.-\frac{72}{(12 k+6)^{2}}-\frac{9}{(12 k+7)^{2}}-\frac{9}{(12 k+8)^{2}}-\frac{5}{(12 k+10)^{2}}+\frac{1}{(12 k+11)^{2}}\right) .
\end{aligned}
$$

Interestingly, there is a corresponding binary formula for $\pi^{2}$ but there is provably no similar decimal formula for $\pi$ [8, Ch. 3].

4. Identification of Multiple Zeta Constants A large number of results has been found over the last 15 years using PSLQ in the course of research on multiple zeta sums, such as those shown in Table 1. After computing the numerical values of these constants, a PSLQ program was used to determine if a given constant satisfied an identity of a conjectured form. These efforts produced numerous empirical evaluations and suggested general results $[2,9]$. Eventually, elegant proofs were found for many of these specific and general results. Three examples of PSLQ results that were subsequently proven are given in Table 1. In the table, $\zeta(t)=\sum_{j=1}^{\infty} j^{-t}$ is the Riemann zeta function, and $\operatorname{Li}_{n}(x)=\sum_{j=1}^{\infty} x^{j} j^{-n}$ denotes the polylogarithm function.

5. Ising integrals. One particularly fruitful application of these methods is the evaluation of definite integrals, such as those that arise in mathematical physics. For example, recently the present authors, together with Richard Crandall, investigated three classes of $n$-fold integrals, which arise in Ising theory and also (in some cases) in quantum field 


$$
\begin{aligned}
\sum_{k=1}^{\infty}\left(1+\frac{1}{2}+\cdots+\frac{1}{k}\right)^{2}(k+1)^{-4}=\frac{37}{22680} \pi^{6}-\zeta^{2}(3) \\
\sum_{k=1}^{\infty}\left(1+\frac{1}{2}+\cdots+\frac{1}{k}\right)^{3}(k+1)^{-6}=\zeta^{3}(3)+\frac{197}{24} \zeta(9)+\frac{1}{2} \pi^{2} \zeta(7) \\
\quad-\frac{11}{120} \pi^{4} \zeta(5)-\frac{37}{7560} \pi^{6} \zeta(3) \\
\sum_{k=1}^{\infty}\left(1-\frac{1}{2}+\cdots+(-1)^{k+1} \frac{1}{k}\right)^{2}(k+1)^{-3}=4 \operatorname{Li}_{5}\left(\frac{1}{2}\right)-\frac{1}{30} \ln ^{5}(2)-\frac{17}{32} \zeta(5) \\
-\frac{11}{720} \pi^{4} \ln (2)+\frac{7}{4} \zeta(3) \ln ^{2}(2)+\frac{1}{18} \pi^{2} \ln ^{3}(2)-\frac{1}{8} \pi^{2} \zeta(3)
\end{aligned}
$$

Table 1: Some multiple zeta identities found by PSLQ

theory:

$$
\begin{aligned}
C_{n} & :=\frac{4}{n !} \int_{0}^{\infty} \cdots \int_{0}^{\infty} \frac{1}{\left(\sum_{j=1}^{n}\left(u_{j}+1 / u_{j}\right)\right)^{2}} \frac{\mathrm{d} u_{1}}{u_{1}} \cdots \frac{\mathrm{d} u_{n}}{u_{n}} \\
D_{n} & :=\frac{4}{n !} \int_{0}^{\infty} \cdots \int_{0}^{\infty} \frac{\prod_{i<j}\left(\frac{u_{i}-u_{j}}{u_{i}+u_{j}}\right)^{2}}{\left(\sum_{j=1}^{n}\left(u_{j}+1 / u_{j}\right)\right)^{2}} \frac{\mathrm{d} u_{1}}{u_{1}} \cdots \frac{\mathrm{d} u_{n}}{u_{n}} \\
E_{n} & =2 \int_{0}^{1} \cdots \int_{0}^{1}\left(\prod_{1 \leq j<k \leq n} \frac{u_{k}-u_{j}}{u_{k}+u_{j}}\right)^{2} \mathrm{~d} t_{2} d t_{3} \cdots \mathrm{d} t_{n},
\end{aligned}
$$

where in the last line $u_{k}=t_{1} t_{2} \cdots t_{k}$.

Computing high-precision values of $n$-fold integrals such as this is very difficult for $n$ greater than three or four. But we a simple substitution reduces a $C$ integral to a 1-dimensional integral:

$$
C_{n}=\frac{2^{n}}{n !} \int_{0}^{\infty} t K_{0}^{n}(p) \mathrm{d} t
$$

where $K(t)$ is the modified Bessel function. In this form, we were able to evaluate $C_{n}$ to over 1000-digit accuracy, for $n$ up to 1024 . With these numerical values in hand, we quickly found that $C_{1}=2, C_{2}=1, C_{3}=L_{-3}(2)=\sum_{n \geq 0}\left(1 /(3 n+1)^{2}-1 /(3 n+2)^{2}\right)$, and $C_{4}=7 \zeta(3) / 12$. We also discovered numerically that

$$
\lim _{n \rightarrow \infty} C_{n}=2 e^{-2 \gamma},
$$

where $\gamma$ is Euler's constant. Further computation established results such as:

$$
D_{2}=1 / 3, \quad D_{3}=8+4 \pi^{2} / 3-27 \mathrm{~L}_{-3}(2), \quad D_{4}=4 \pi^{2} / 9-1 / 6-7 \zeta(3) / 2
$$

and

$$
\begin{aligned}
E_{2}= & 6-8 \log 2, \quad E_{3}=10-2 \pi^{2}-8 \log 2+32 \log ^{2} 2 \\
E_{4}= & 22-82 \zeta(3)-24 \log 2+176 \log ^{2} 2-256\left(\log ^{3} 2\right) / 3 \\
& +16 \pi^{2} \log 2-22 \pi^{2} / 3 \\
E_{5} \stackrel{?}{=} & 42-1984 \operatorname{Li}_{4}(1 / 2)+189 \pi^{4} / 10-74 \zeta(3)-1272 \zeta(3) \log 2 \\
& +40 \pi^{2} \log ^{2} 2-62 \pi^{2} / 3+40\left(\pi^{2} \log 2\right) / 3+88 \log ^{4} 2 \\
& +464 \log ^{2} 2-40 \log 2 .
\end{aligned}
$$


The $E_{5}$ integral was found after transforming its defining 5 -fold integral representation into an extremely complicated 3-fold integral. We then computed this 3 -fold integral to 250-digit precision, by using a parallel quadrature program implemented on 1024 CPUs of a parallel computer system, and then discovered the above-listed experimental identity by using PSLQ. This identity has a question mark because, unlike the others mentioned in this paper, we do not yet have a formal proof. Nonetheless it is established numerically at least 180 orders of magnitude beyond the level of numerical "chance," and so we are quite confident in the result. Such confidence is typically obtainable if the constants involved can be computed to sufficiently high precision. Sometimes as with $C_{n}$ this is relatively easy. In other cases, such as $E_{5}$, it involves much more labor.

\section{References}

[1] David H. Bailey, "A Compendium of BBP-Type Formulas," available at http://crd.lbl.gov/ dhbailey/dhbpapers/bbp-formulas.pdf.

[2] David H. Bailey, Jonathan M. Borwein and Roland Girgensohn, "Experimental Evaluation of Euler Sums," Experimental Mathematics, vol. 4, no. 1, 1994, pg. 17-30.

[3] David H. Bailey, Peter B. Borwein and Simon Plouffe, "On The Rapid Computation of Various Polylogarithmic Constants," Math. of Computation, vol. 66, no. 218, 1997, pg. 903-913.

[4] David H. Bailey and David J. Broadhurst, "Parallel Integer Relation Detection: Techniques and Applications," Math. of Computation, vol. 70, no. 236, pg. 1719-1736.

[5] David H. Bailey, J. M. Borwein and R. E. Crandall, "Integrals of the Ising class," Journal of Physics A: Mathematical and General, vol. 39 (2006), pg. 12271-12302.

[6] David H. Bailey, Jonathan M. Borwein, Vishal Kapoor and Eric Weisstein, "Ten Problems in Experimental Mathematics," American Math. Monthly, vol. 113, no. 6, 2006, pg. 481-409.

[7] David H. Bailey, Yozo Hida, Xiaoye S. Li and Brandon Thompson, "ARPREC: An Arbitrary Precision Computation Package," Sept. 2002, available at http://crd.lbl.gov/ dhbailey/dhbpapers/arprec.pdf.

[8] Jonathan M. Borwein and David H. Bailey, Mathematics by Experiment, AK Peters, 2003. Second edition, 2008. See also http://www. experimentalmath.info.

[9] David Borwein, Jonathan M. Borwein and Roland Girgensohn, "Explicit Evaluation of Euler Sums," Proc. Edinburgh Math. Society, vol. 38, 1995, pg. 277-294.

[10] Helaman R. P. Ferguson, David H. Bailey and Stephen Arno, "Analysis of PSLQ, An Integer Relation Finding Algorithm," Math. of Computation, vol. 68, 1999, pg. 351-369. 